\title{
Perennización de las precisiones de la faceta auricular del hueso ilion a través de réplicas óseas en silicona y yeso para estudios antropológicos forenses
}

\author{
José Yván Vargas Bourguet \\ jose.vargas@untrm.edu.pe \\ https://orcid.org/0000-0002-6031-8585 \\ Universidad Nacional Toribio Rodríguez de Mendoza de Amazonas, \\ Perú
}

\section{RESUMEN}

El presente artículo pretende aproximarse al proceso de perennización de las precisiones o particulares que exponen las piezas óseas durante el análisis del perfil bioantropológico de un individuo. Para este estudio, se utilizaron los originales de las facetas auriculares del hueso ilion derecho e izquierdo de un individuo de sexo masculino y con una edad al momento de la muerte de entre 30 a 34 años, de acuerdo al método propuesto por Lovejoy et al. (1985), en donde los resultados permitieron obtener réplicas óseas que expresan una valorización importante de fidelidad en relación a las originales tanto en su morfología, estructura y precisiones de acuerdo a los cambios en la faceta auricular que corresponde a la fase III del método de Lovejoy et al. (1985). Para la elaboración de estas réplicas óseas, se aplicó una técnica de moldeados en base a silicona y yeso de fácil acceso y de bajo costo respecto a otros insumos o materiales de reproducción de impresiones óseas, también, es importante precisar que, este procedimiento de replicas óseas puede ser útil como una herramienta didáctica en los procesos de enseñanza-aprendizaje en los estudiantes y profesionales de antropología forense.

Palabras clave: réplica; hueso ilion; antropología; forense; silicona. 


\title{
Perennialization of the precisions of the auricular facet of the ilium bone through bone replicas in silicone and plaster for forensic anthropological studies
}

\begin{abstract}
This article aims to approach the process of perennialization of the details or particulars that bone pieces expose during the analysis of the bioanthropological profile of an individual. For this study, the originals of the auricular facets of the right and left hip bone of a male individual and with an age at death between 30 to 34 years were used, according to the method proposed by Lovejoy et al. (1985), where the results allowed to obtain bone replicas that express an important valuation of fidelity in relation to the originals both in their morphology, structure and precisions according to the changes in the auricular facet that corresponds to phase III of the method of Lovejoy et al. (1985). For the elaboration of these bone replicas, a technique of molding based on silicone and plaster of easy access and low cost was applied compared to other supplies or materials for the reproduction of bone impressions, it is also important to specify that, this procedure of replicas bone can be useful as a didactic tool in the teaching-learning processes in forensic anthropology students and professionals.
\end{abstract}

Keywords: replica, hip bone, anthropology, forensic, silicone.

Artículo recibido: 10 Setiembre. 2021 Aceptado para publicación: 15 Octubre. 2021 Correspondencia: jose.vargas@untrm.edu.pe Conflictos de Interés: Ninguna que declarar 


\section{INTRODUCCIÓN}

Durante el análisis de la faceta o superficie auricular del hueso ilion, el antropólogo forense puede registrar y documentar información valiosa para estimar la edad de un individuo adulto esqueletizado aplicando el método de Lovejoy $(1985)^{1}$ para un contexto de población mestiza, como es el caso peruano. Si bien es cierto que el uso de la faceta auricular presenta mayor conservación que la región púbica, de acuerdo con el análisis de colecciones arqueológicas (Lovejoy et al., 1985; Meindl y Lovejoy, 1989) no siempre estos indicadores se muestren conservados. En algunos escenarios, la superficie auricular y la sínfisis pública pueden resultar frágiles (Villarroel y Medina, 2011) por lo tanto rompibles durante su manipulación, esto debido a los procesos de remodelación ósea (Gutiérrez, 2008), procesos patológicos (Von Endt y Ortner, 1984), degenerativos, perimortem o tafonómicos que ha sufrido el cadáver y que por lo tanto han alterado al hueso, corriendo el riesgo de perder información relevante.

Así mismo, una vez que se ha finalizado los estudios del perfil biológico de restos óseos en los laboratorios de antropología, estos son remitidos o devueltos a los operadores de justicia para la entrega final respectiva a los familiares, y en otros casos, para ser inhumados, quedando perennizado estas muestras óseas a un nivel fotográfico y fílmico en el mejor de los casos. Esta forma de perennización limita a que estas muestras óseas puedan ser estudiadas posteriormente en su contexto espacial y en 3D (D'Acuña y Guiraldes, 1998) dificultando el adecuado análisis de la estructura ósea (Villarroel y Medina, 2011). A ello se suma, el hecho de que la adquisición de muestras óseas reales por parte de los laboratorios de antropología física forense de las universidades presentan serias dificultades para los estudios de carácter académico o científico, inclusive por parte de los mismos estudiantes en antropología al acceso de éstos en los laboratorios forenses de las instituciones públicas del Estado, como el Instituto de Medicina Legal y Ciencias Forenses en el contexto peruano, el cual es restringido su acceso por la rigurosidad de la normativa existente. Y en lo mejor de los casos, la manipulación de restos óseos por parte de los estudiantes se da únicamente al interior de los laboratorios (Delgado et al. 2009) por los riesgos que se acarrea (biológicos, extravío de piezas óseas, etc.)

\footnotetext{
${ }^{1}$ Lovejoy y et al. (1985) realizaron un estudio en la faceta auricular en donde propusieron un método para estimar la edad biológica en función a cinco aspectos de la estructura de estas facetas, estableciendo cambios importantes asociados a la edad en ocho etapas con rangos que van desde los 20 hasta los 60 años de edad (Ramey, 2008).
} 
Por otro lado, es de conocimiento que el Estado peruano tiene regulado ciertas normas a cumplir para que un cadáver sea utilizado para fines académicos científicos, tal como lo estipula el artículo $49^{\circ}$ del Reglamento de la Ley de Cementerios y Servicios Funerarios, al sostener que cuando los cadáveres no son reclamados en el plazo de 48 horas pueden ser inhumados y sean destinados para fines de investigación científica, y en los casos en donde el cadáver haya sido donado por voluntad expresa del familiar del occiso (Decreto Supremo $\mathrm{N}^{\circ}$ 03-94-SA). Cumplir con este proceso normativo dificulta el acceso inmediato y oportuno para los estudios científicos de manipulación de cadáveres en los laboratorios de antropología física de las universidades. Se suma a ello las limitaciones en su conservación y los problemas biológicos que se puedan generar (Quijano, Rodríguez, Peralta, Cortés, 2012).

En ese orden de ideas, es que se ha adoptado la técnica de perennización de las precisiones de la faceta auricular del hueso ilion a través de réplicas óseas empleando materiales como silicona de consistencia fluida y de condensación de baja y de alta viscosidad, catalizador en gel, yeso piedra extraduro y agua para estudios antropológicos forenses. Nos resulta de vital importancia el realizar una perennización o reproducción ósea de individuos analizado en laboratorio cada vez que se ha tenido la oportunidad en su manipulación, a través de la réplica de estos, utilizando para ello estos materiales que permita sobre todo conservar las características o indicadores que conllevan a determinar y estimar el perfil bioantropológico.

Las investigaciones sobre el uso de materiales de adición como siliconas y resinas en la fabricación de materiales anatómicos son diversos (Quijano, Rodríguez, Peralta, Cortés, 2012; Villarroel y Medina, 2011; Matamala et al., 1988; Oda \& Sant'Ana, 2003). La silicona es un compuesto que actúa como un polímero y que reacciona gracias a un catalizador químico el cual éste aporta versatilidad en los mecanismos de impresión, y que pueden ser de consistencia fluida y de condensación baja o alta en viscosidad (Banchieri, 2016). La polimerización por adición no conforma subproductos (López, Rodríguez, Espinoza, 2018), por lo tanto, la silicona se convierte en un elemento con estabilidad a nivel de dimensiones y de uso casi perfecta durante una impresión (Ramírez, 2017). Por otro lado, el empleo del yeso piedra extraduro hidratado es de gran utilizad cada vez que se pretende producir doble impresión. En ese sentido, todos estos insumos nos permiten registrar información a nivel del tejido blando como en huesos permitiendo 
la reproducción de réplicas anatómicas.

El uso de estos materiales es de común utilidad y exposición en el campo de la odontología sobre todo en la elaboración de prótesis (López, Rodríguez, Espinoza, 2018). Estos materiales al ser trasladados a los laboratorios de antropología para ser empleados en la elaboración de réplicas de muestras óseas implican costos económicos bajos (Villarroel y Medina, 2011) en su adquisición, así mismo el acceso a estos materiales no es restringido, siendo de fundamental importancia y de interés antropológico forense para fines pedagógicos y andragógicos.

En la presente investigación se intenta demostrar el procedimiento a seguir para la obtención de replicas o reproducciones óseas, específicamente de las precisiones o particularidades que expone la faceta auricular de la región pélvica, creando un molde primario y secundario que permita poder replicar estas muestras óseas cada vez que se requiera y de esa manera poder perennizar las precisiones de los cambios y variabilidades relacionados con la edad para posteriores estudios académicos e investigaciones científicas en el campo de la antropología forense.

Considerando que esta investigación es apenas un corto paso en este tipo de ejercicios didácticos forenses que puede servir como una alternativa para reemplazar las muestras óseas reales cada vez que se tenga la ocasión evitando así el deterioro de piezas óseas, se insta a los interesados a continuar con estas técnicas para su mejoramiento, convencidos que puede ser de mucha utilidad en la perennización de otras precisiones o características óseas en la estimación de la edad en adultos (sínfisis púbica, cambios en el cráneo, metamorfosis de los fines esternales de las costillas y modificación en las vértebras), o características relacionadas al sexo biológico, lateralidad, estatura e inclusive en la perennización de fracturas donde se evidencien cicatrización o remodelación en la superficie del hueso, así como aquellas marcas lesionales asociadas a mecanismo de producción de fracturas en contexto perimortem.

\section{MATERIAL Y MÉTODOS}

La técnica puesta en práctica ha exigido la utilización de los siguientes materiales:

Silicona de consistencia fluida de condensación de baja viscosidad (Oranwash L), silicona de consistencia fluida de condensación de alta viscosidad (Zetaplus), catalizador en gel (Indurent gel, de color rojo para facilitar la visualización de la mezcla homogénea), yeso 
piedra extraduro y agua (medio vaso). Las muestras óseas empleadas como modelo a reproducir fueron: la faceta auricular del ilion derecho e izquierdo de la pelvis.

Las muestras óseas utilizadas pertenecen a la colección osteológica del Área de Antropología Forense de la Unidad Médico Legal de la ciudad de Moyobamba en el Departamento de San Martín - Perú, en esta oportunidad se ha tenido a disposición ambos iliones en su estado completos de un individuo el cual se ha protegido su identidad durante todo el proceso de la investigación. Sin embargo, estos iliones corresponden a un individuo de sexo biológico masculino, así como su edad corresponde a la fase III de acuerdo al método de Lovejoy (1985) en el análisis de la faceta auricular, con un rango de edad entre 30 a 34 años.

Para una correcta réplica de piezas óseas es necesario considerar la buena selección y extracción del mismo (Villarroel y Medina, 2011) es por ello que ambas piezas óseas fueron seleccionadas tomando en cuenta el adecuado estado de conservación y limpieza para obtener de esta manera un óptimo modelo.

Para obtener el primer molde, se seleccionaron la superficie o faceta auricular de los iliones derechos e izquierdo tomando en cuenta el adecuado estado de conservación, para una correcta replica de piezas óseas (Villarroel y Medina, 2011) y por consiguiente obtener un óptimo modelo (Fig. 1).

En un segundo momento se precedió a delimitar la región a moldear (zona de impresión) utilizando un lápiz de color para resaltar el borde.

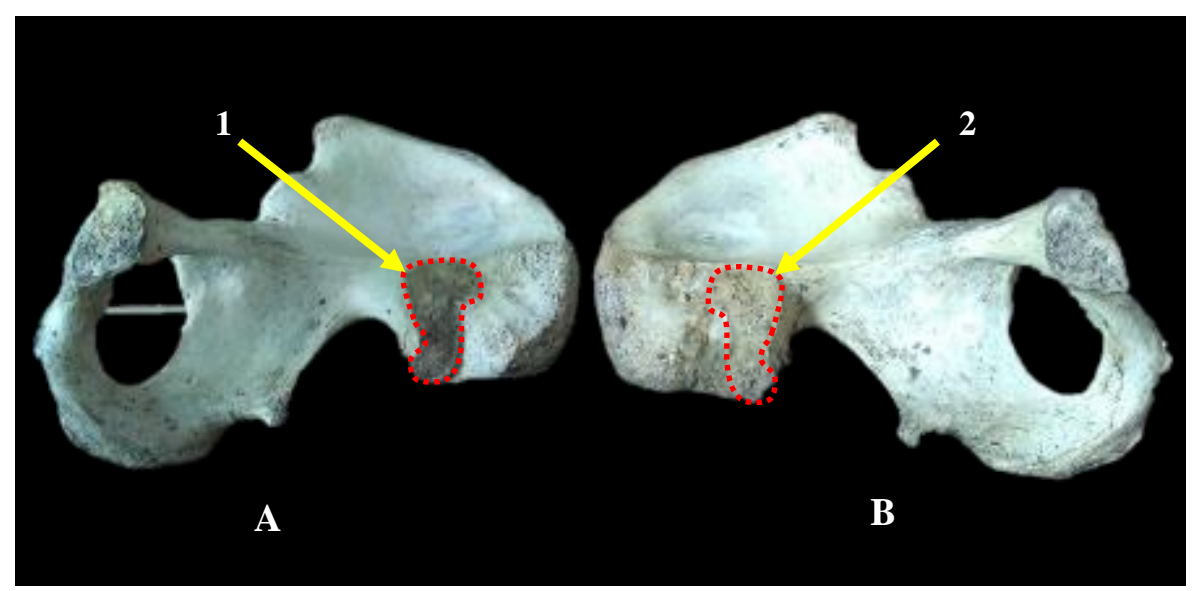

Figura 1. Muestras óseas seleccionadas y utilizadas. A) Hueso ilion derecho. (1) Faceta auricular. B) Hueso ilion izquierdo. (2) Faceta auricular. 
El tercer paso consistió en la preparación de la mezcla para el primer molde, en donde se combinó la silicona de consistencia fluida y de condensación de baja viscosidad con el activador catalizador en gel (color rojo). La cantidad de esta mezcla dependió de la superficie del modelo a replicar. En este caso, para las dos facetas auriculares la proporción de silicona que se utilizó fue $10 \mathrm{~mL}$ (el equivalente a dos cucharaditas), mientras que el catalizador en una proporción de $5 \mathrm{~mL}$. Este tipo de silicona debido a su baja viscosidad ofrece una mejor impresión en la precisión de los detalles que se desea imprimir en la superficie. Mientras que el catalizador aporta una mejor versatilidad de uso en las diferentes técnicas de impresión y su color rojo nos facilita la mejor visualización de la mezcla homogénea.

Acto seguido, se procedió a transferir la mezcla sobre ambas facetas auriculares (Fig. 2), dejando secar hasta que pierda su estado pegajoso.
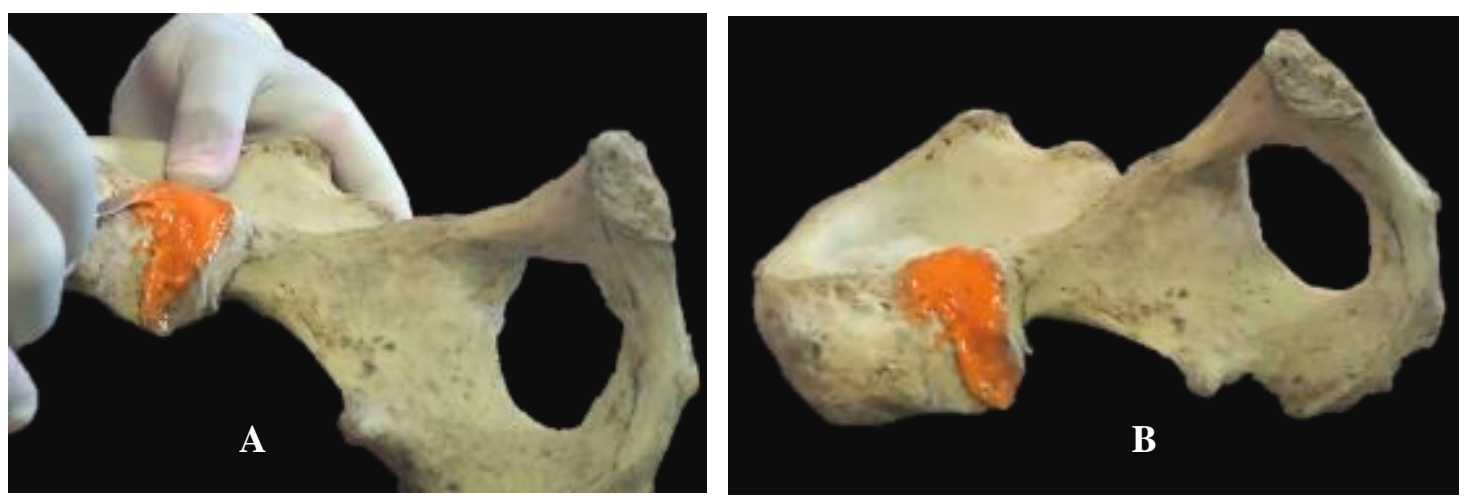

Figura 2. Vista general del hueso ilion izquierdo. A) Aplicación de la mezcla primaria sobre la superficie auricular. B) Superficie auricular durante el secado, el cual oscila entre 30 minutos a una hora de tiempo.

En un quinto momento, se preparó la segunda mezcla, en este caso entre la silicona más pesada conocido como zetaplus, el cual es de consistencia fluida y de condensación de alta viscosidad con el mismo activador que se utilizó en la mezcla inicial, generando una masa (similar a la textura de una plastilina). La proporción que se utilizó fue de 80 gramos (equivalente a $1 / 2$ taza aproximadamente), mientras cantidad del catalizador fue de $5 \mathrm{~mL}$ (el equivalente a una cucharadita). Este tipo de silicona, nos ofrece mayor resistencia a una fractura por su dureza al final del secado.

Posteriormente, como sexto paso, se procedió aplicar esta segunda combinación sobre la primera mezcla (Fig. 3) el cual reposa a su vez sobre la superficie auricular. Se dejó secar aproximadamente por 30 minutos. Una vez que la silicona se ha activado, se realizó el 
retiro de la superficie auricular, ayudándonos para esto de una espátula quirúrgica de cuidadosa. De esta manera se obtuvo el molde primario.
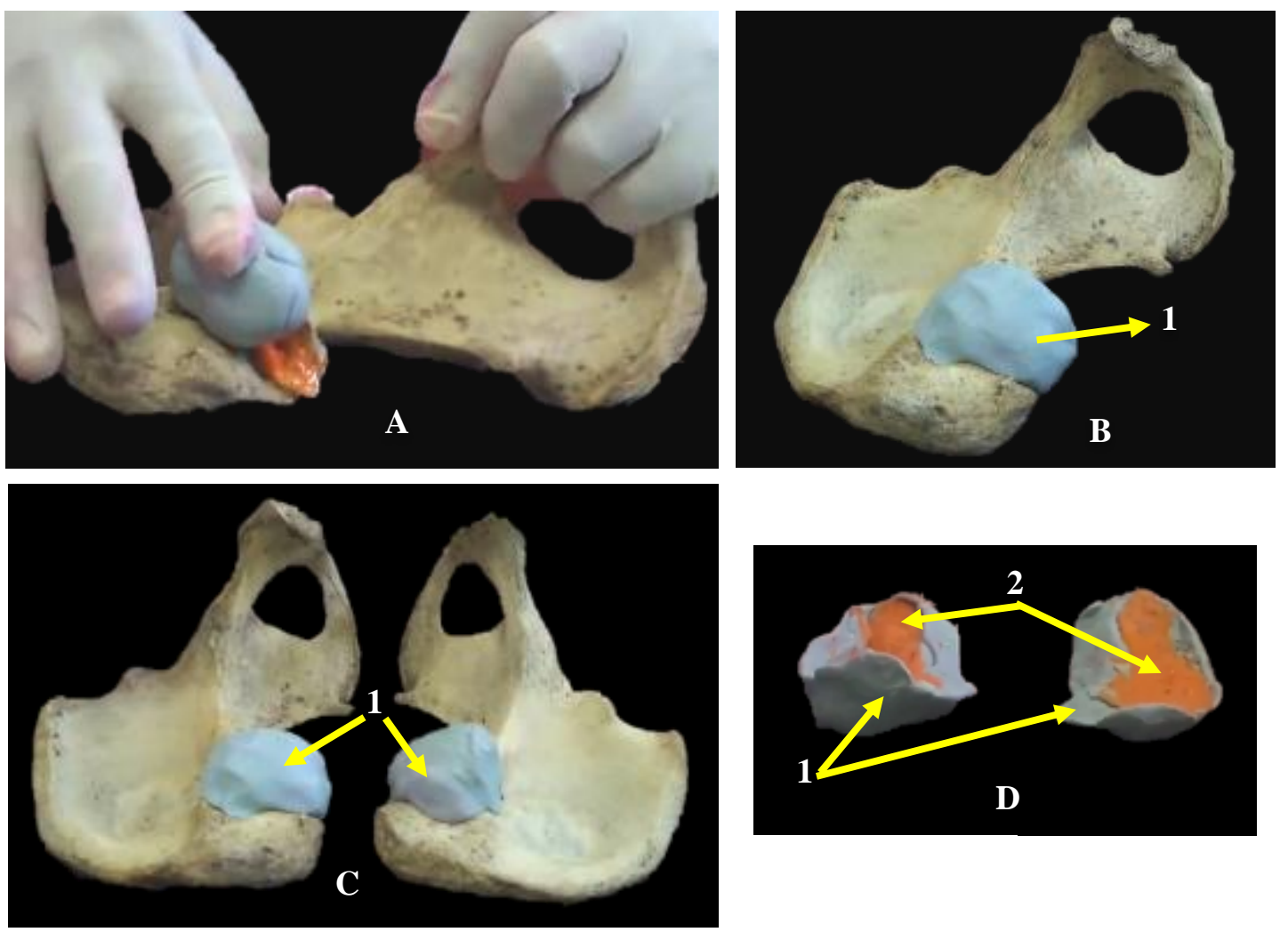

Figura 3. A) Aplicación de la segunda mezcla sobre la primera mezcla. B) Vista del ilion izquierdo. (1) Masa de la mezcla secundaria sobre la superficie auricular. C) Vista general del ilion derecho e izquierdo. (1) Masa de mezcla secundaria transferida completamente sobre la superficie auricular durante secado. D). Molde primario. (1) Mezcla secundaria compacta, sólida y dura compuesta por silicona fluida de alta viscosidad y catalizador de gran versatilidad. (2) Mezcla primaria compacta compuesta por silicona fluida de baja viscosidad y catalizador de gran versatilidad, en donde se ha registrado las impresiones de las características o precisiones de la superficie auricular de los huesos iliones, el cual servirá de molde

El paso siete, correspondió a la elaboración del segundo molde, para ello se preparó una tercera mezcla utilizando el yeso piedra extraduro el cual fue hidratado con proporciones adecuadas de agua hasta generar una de textura espesa. Es necesario tener en cuenta que el adicionar demasiado yeso podemos incurrir en una mezcla demasiado débil, corriendo el riesgo que las precisiones del primer moldeado desaparezcan. Para ello se ha utilizado las siguientes proporciones: cinco (5) cucharadas de yeso y dos (2) cucharadas de agua, el cual han sido suficientes para los dos moldes primarios (moldeado de la faceta auricular derecha e izquierda). La proporción de agua fue en relación al espesor que se logró 
producir con el yeso. Luego, esta tercera mezcla se vertió en estado denso o espeso en los moldes primarios, realizando ligeros vibrados en el molde primario para que la mezcla se adicione en toda su superficie. Se dejó secar por el tiempo de dos horas. (Fig. 4). Posterior a ello, se extrajo el molde secundario compactado y duro, expresandose la réplica de la faceta auricular de los iliones.
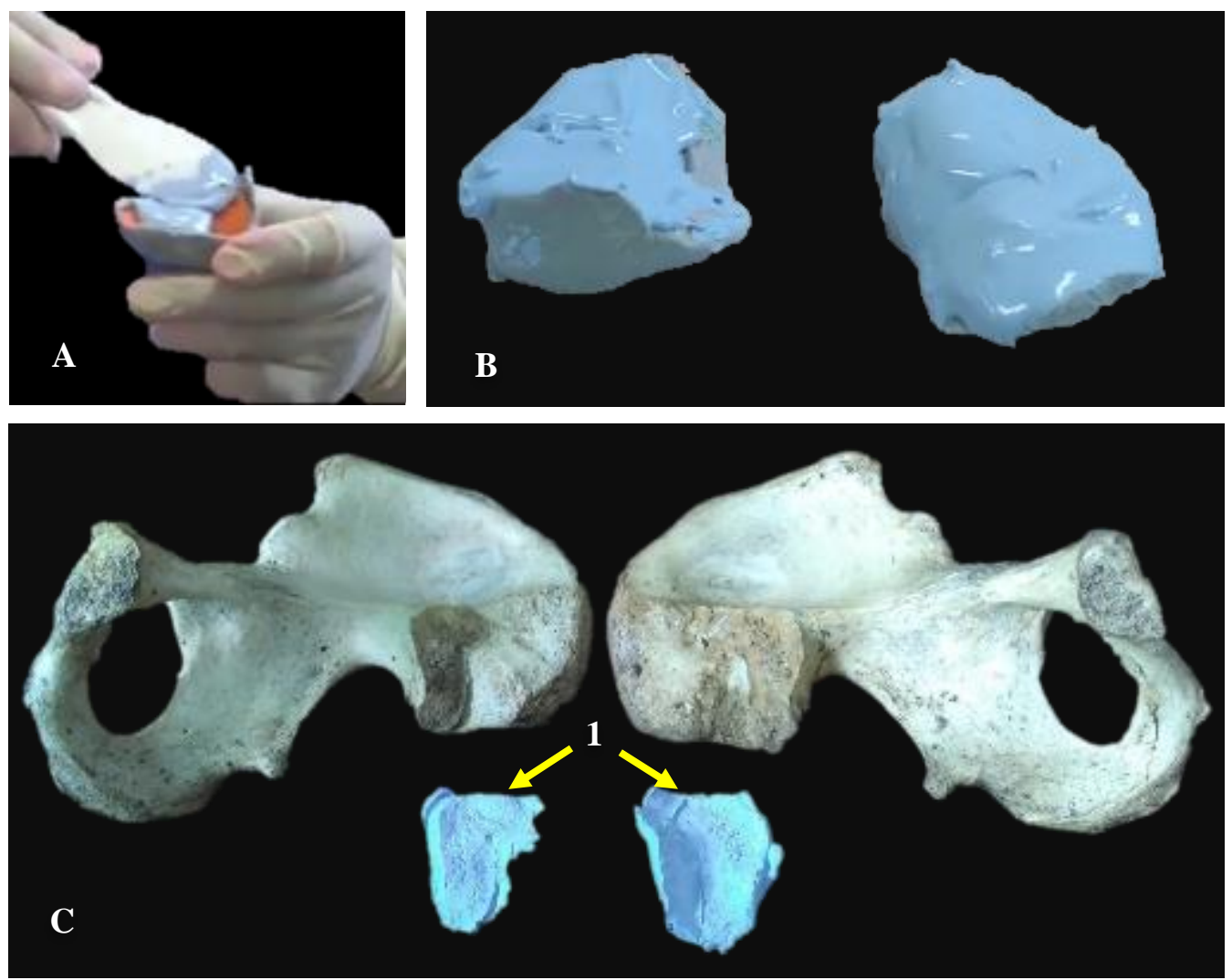

Figura 4. A) Aplicación de la tercera mezcla sobre el primer moldeado. B) Vista la tercera mezcla sobre el primer moldeado (izquierdo y derecho). C) Vista general del hueso ilion derecho (imagen superior de la izquierda) y ilion izquierdo (imagen superior de la derecha). (1) Resultado final de réplicas óseas de las facetas auriculares reproducidas.

Para retirar las excrecencias de yeso formado en los bordes de los moldes finales, se utilizaron herramientas quirurjicas como la tijera para escayola y la sierra electrica corta yeso el cual nos permitieron eliminar los elementos innecesarios y obtener las réplicas oseas adecuadas. 


\section{RESULTADOS}

Las réplicas óseas presentaron las precisiones o características de las facetas auriculares de los huesos iliones derecho e izquierdo con un valor importante de exactitud.

Durante el examen comparativo entre los huesos originales con sus respectivas reproducciones, se resaltan las particularidades en la morfología, estructura y las precisiones que se exponen en la superficie auricular presentando un importante grado de fidelidad.

Es así que la replica de hueso de la faceta auricular derecha e izquierda permite observar su morfología estructural resaltando: en la réplica de la faceta auricular derecha, en su vista superior, se observa la semicara superior (1) que se ubica en la parte superior de la línea del ápice, mientras que la semicara inferior (4) se reconoce en la parte inferior, ambas semicaras constituyen la morfología general de la superficie auricular que presenta una apariencia de un boomerang con el ápice (3) el cual comprende a la parte del contorno de la articulación en contacto inmediato con la parte final y posterior de la línea arcuata (2) el cual es una línea imaginaria de trayectoria arqueada o semilunar por encima de la superficie interna y posterolateral de hueso ilión. (Fig. 5).
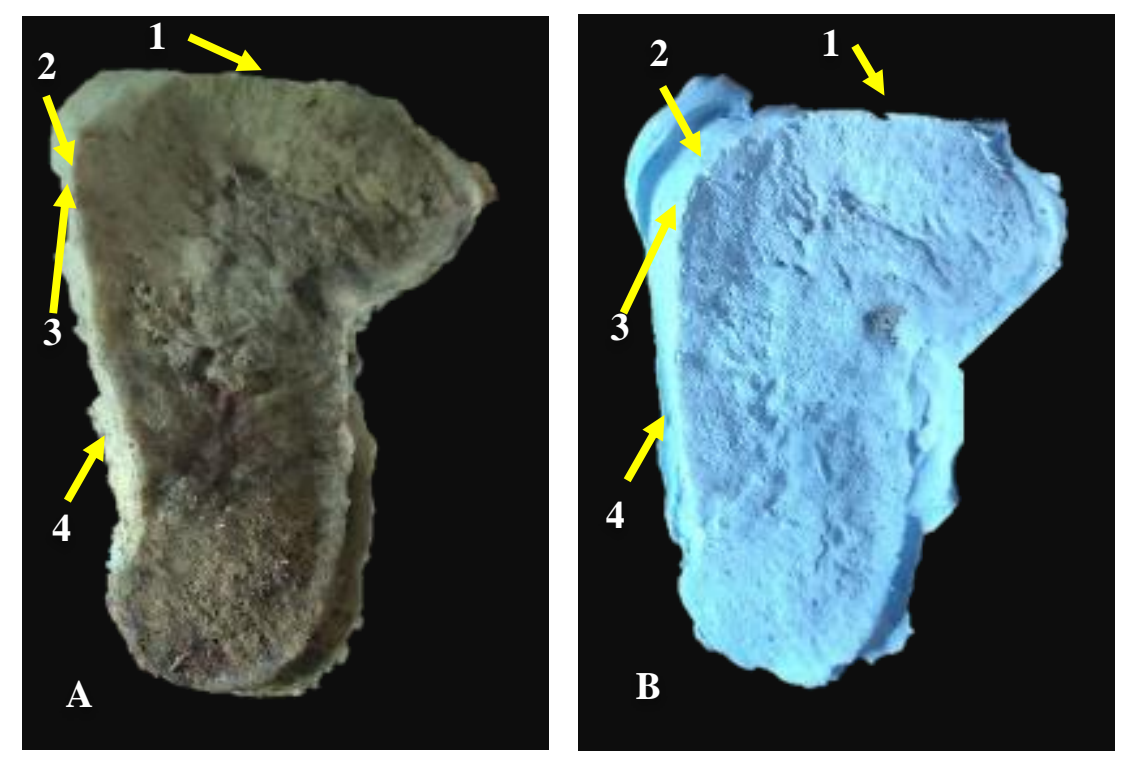

Figura 5. Faceta auricular derecha del hueso ilion, vista posterolateral. A) Pieza original y B) Réplica final. (1) Semicara superior; (2) Línea arcuata; (3) Ápice y (4) Semicara inferior.

La réplica del hueso de la faceta auricular izquierda, de igual forma que la réplica anterior, presenta en su vista superior los procesos de su morfología correspondiente a: una semicara superior (1) que se ubica en la parte superior de la línea del ápice, mientras que 
la semicara inferior (4) se reconoce en la parte inferior, ambas semicaras constituyen la morfología general de la superficie auricular que presenta una apariencia de un boomerang con el ápice (3) el cual comprende a la parte del contorno de la articulación en contacto inmediato con la parte final y posterior de la línea arcuata (2) el cual es una línea imaginaria de trayectoria arqueada o semilunar por encima de la superficie interna y posterolateral de hueso ilión. (Fig. 6).
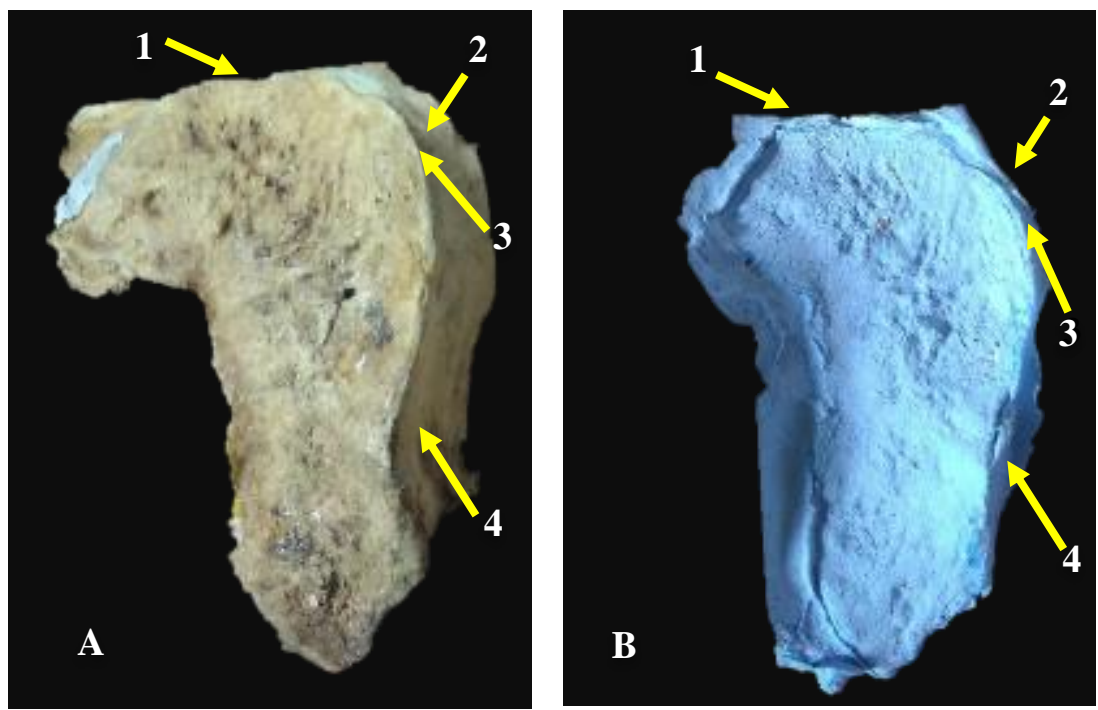

Figura 6. Faceta auricular izquierda del hueso ilion, vista posterolateral. A) Pieza original y B) Réplica final. (1) Semicara superior; (2) Línea arcuata; (3) Ápice y (4) Semicara inferior.

Respecto a las precisiones o particularidades que se expone en las facetas auriculares de los huesos originales, han sido reproducidas notablemente en las réplicas óseas y que permite visualizar sin dificultad las modificaciones establecidas en las facetas auricular para estimar la edad correspondiente y que de acuerdo al método de Lovejoy (1985) se tienen que establecer características particulares para estimar una edad, en este caso de la fase III (30 a 34 años de edad). Es así que en una vista posterolateral de la pieza replicada (Fig. 7) se puede apreciar en la superficie auricular derecha e izquierda débil presencia ondulaciones (1), con ligeras exposiciones de finas estrías (2). Así mismo, se puede evidenciar granulación en la parte anterior de la superficie (3), mientras que el ápice se encuentra aún bien definido (4), las cuales han ido reproducidas fielmente de las piezas originales. 

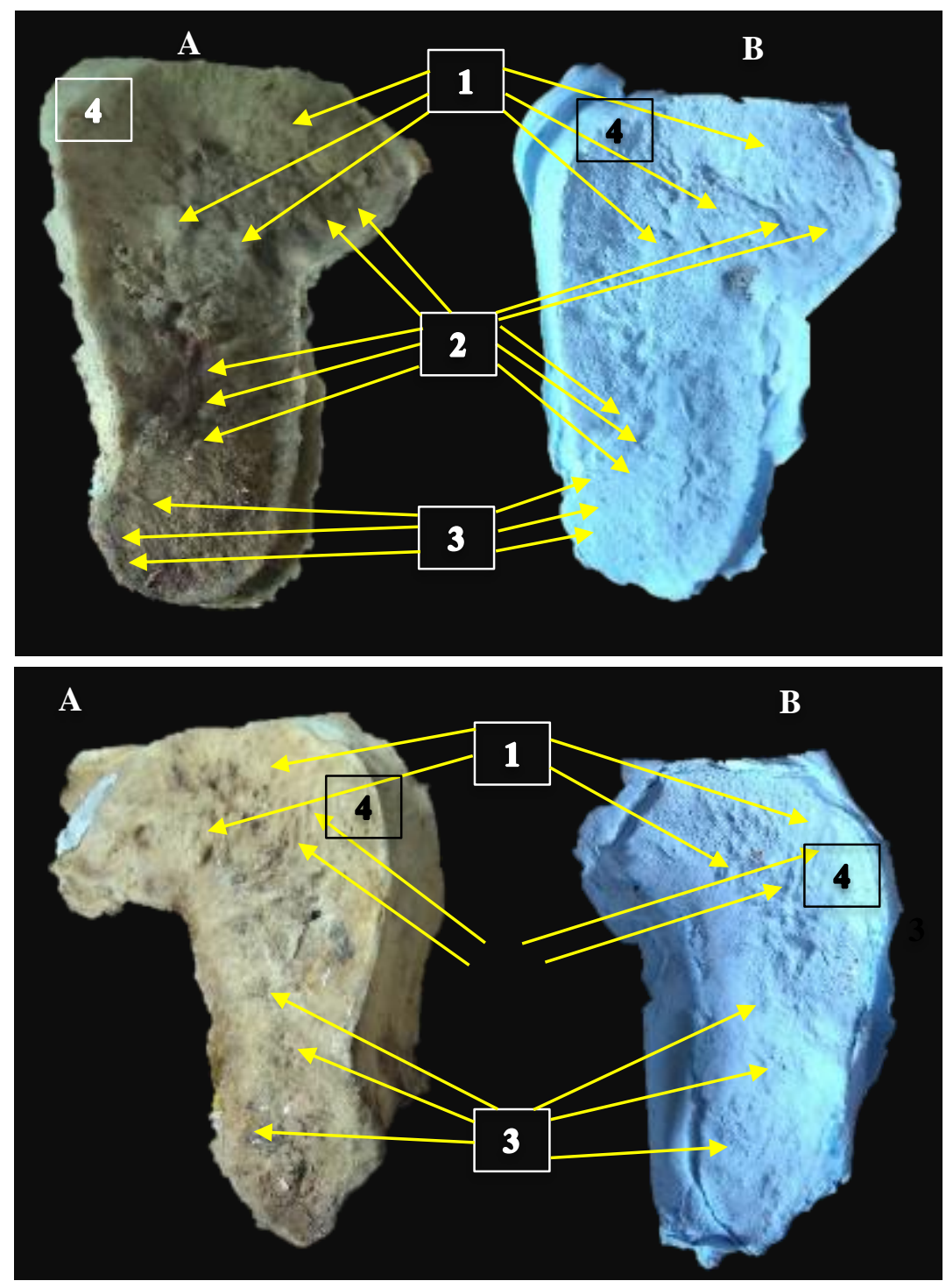

Figura 7. Facetas auriculares lado derecho original (A) y réplica (B) (imágenes superiores). Facetas auriculares lado izquierdo original (A) y replica (B) (imágenes inferiores). (1) Presencia débil de ondulaciones; (2) Exposición de finas estrías; (3) Visualización de granulaciones y (4) Ápice sin cambios significativos.

\section{DISCUSIÓN}

En los procesos de transmisión de conocimientos y de aprenderlos, la manipulación de materiales didácticos como los moldeados que conservan fielmente las características tanto en su forma y precisiones anatómicas son recursos muy seguros en estos procesos (Segovia \& Moreno, 1989) cada vez que se siga con los procedimientos técnicos en la elaboración de réplicas anatómicas. 
Al igual que en la mayoría de investigaciones de reproducción de piezas óseas, la orientación de esta propuesta de reproducción consistió en el manejo reducido de materiales o insumos, evitando las posibles alteraciones en el hueso durante su manipulación (Prevedorou y Buikstra, 2019), así como también minorar los costos en la adquisición de estos insumos de reproducciones anatómicas (Kurser, 2006; Villarroel, Medina, 2011; Quijano, Rodríguez, Peralta, Cortéz 2012).

Con el uso de la técnica propuesta podemos obtener reproducciones oseas con tal fidelidad en sus dimensiones tridimencionales (Villarroel y Medina 2011) en su morfología y precisiones oseas que nos permite visualizar los cambios en su superficie auricular como por ejemplo la porosidad, granulosidad, odulaciones, estrillas y densidad que vienen hacer las madificiaciones que se observan en la estructura de la faceta auricular de acuerdo a los procesos de cambio asociados a la edad.

Esta investigacion tiene como objetivo presentar una técnica mas sencilla en uso (sin riesgo biológico y con durabilidad) y adquisicion de materiales de este tipo para la reproduccion de piezas oseas y que a su vez pueda servir como una herramienta a los estudiantes y profesionales de antropología con interes forense, para el alcance de sus metas académicas e investigaciones cientificas.

\section{CONCLUSIÓN}

La reproducción ósea de la faceta auricular del ilion derecho e izquierdo resultaron con un grado de fidelidad importante en relación a las piezas óseas originales favorable en su perennización y por lo tanto útil en el proceso de enseñanza-aprendizaje en el campo antropológico forense, convirtiéndose en un recurso didáctico de fabricación sencilla y accesible. Resaltando que esta técnica se encuentra en constante construccion y perfecionamiento y que al ser empleado debe tomarse en cuenta los criterios y prioridades de cada escenario.

\section{REFERENCIAS BIBLIOGRAFICAS}

Banchieri D. (2016). Materiales dentales. Módulo 1. Manual de Apoyo Teórico. Uruguay. Disponible en: https://www.colibri.udelar.edu.uy/bitstream/123456789/8441/1/Librillo\%20Mat eriales\%20Dentales\%201.pdf

Delgado-Rosas, M.; Gorrostieta-Hurtado, E.; Ramos-Arreguín, J.M.; Pedraza-Ortega, J.C.; Aceves-Fernández, M.A. (20099. Obtención de Modelos en resina epóxica 
de un fémur. Memorias del XV Congreso Internacional Anual de la SOMIM, Cd. Obregón, Sonora, septiembre 2009. 10p. Disponible desde Internet en: http://somim.org.mx/ articulos2010/memorias/memorias2009/pdfs/A1/ A1_83.pdf.

D’acuña, E.; Guiraldes Del C., H. 1998. Anatomía del cráneo en páginas Web. Rev. Chil. Anat. 16(1):115-166.

Gutiérrez, J. (jul-sep de 2008). El proceso de remodelación ósea. Medigraphic, 4(3).

Kurzer, M. Estudio comparativo de dureza en dientes artificiales fabricados con diferentes tipos de resinas acrílicas. Revista EIA, 6:121-8, 2006.

López, L.; Rodríguez, D.; Espinoza, N.; (2018). Materiales de impresión de uso estomatológico. Órgano científico estudiantil de ciencias médicas de Cuba. 16 de Abril. 2018;57(267):64-72. http://www.rev16deabril.sld.cu. ISSN:17296935/RPNS:1804

Matamala, F.; Olave, E.; Henríquez, J. \& Chavez, R. (1988). Experiencia de siete años en el Diseño y uso de material didáctico anatómico de bajo costo. An. Anat. Norm., 6(6):208-13.

Meindl R, Lovejoy C.O., Mensforth R.P. \& L.D. Carlos (1985) Accuracy and direction of error in the sexing of the skeleton. American Journal of Physical Anthropology.

Oda, J. \& Sant'Ana, D. Técnica de inclusão em resina de cortes transversais da medula espinhal coradas pelo método de mulligan. Int. J. Morphol., 21(1):49-92, 2003.

Prevedorou, E. A. y Buikstra, J. E. (2019). Bioarchaeological Practice and the Curation of Human Skeletal Remains in a Greek Context: The Phaleron Cemetery. Advances in Archaeological Practice, 7(1), 60-64.

Quijano, Y., Rodríguez, F., Peralta, K., Cortéz, S. (2012). Réplicas óseas en resina poliester como herramienta didáctica para el aprendizaje de anatomía. Rev. U.D.C.A Act. \& Div. Cient. 15(2): 275 - 281, 2012.

Ramey, K. (2008). Manual de Antropología Forense. Ediciones Bellaterra - Barcelona.

Ramírez, JM. (2017). Materiales de impresión odontológico: las siliconas. Colombia; Disponible en: http://www.redencol.com.co/materiales-de-impresionodontologico-las-siliconas/

Segovia, J.; Moreno, E. (1989). Uso de yeso emparafinado en la fabricación de modelos anatómicos. An. Anat. Norm., 7:158-9, 1989. 
Villarroel, M., Medina, E. (2011). Técnica anatómica para restaurar y/o reproducir piezas óseas humanas de dificil obtención, para la investigación y docencia científica. Int. J. Morphol., 29(2):532-536.

Von Endt, D. W. y Ortner, D. J. (1984). Experimental Effects of Bone Size and Temperature on Bone Diagenesis. Journal of Archaeological Science, 11, 247253. 